\title{
Configuration Mixing in Pt Nuclei within Interacting Boson Model-2
}

\author{
Malek A. H. Muhi, Saad N. Abood \\ Physics Department, College of Science, Al-Nahrain University, Baghdad, Iraq \\ Email: malek.muhi@gmail.com
}

How to cite this paper: Muhi, M.A.H. and Abood, S.N. (2020) Configuration Mixing in $\mathrm{Pt}$ Nuclei within Interacting Boson Model-2. Journal of Applied Mathematics and Physics, 8, 492-506.

https://doi.org/10.4236/jamp.2020.83039

Received: January 7, 2020

Accepted: March 10, 2020

Published: March 13, 2020

Copyright $\odot 2020$ by author(s) and Scientific Research Publishing Inc. This work is licensed under the Creative Commons Attribution International License (CC BY 4.0).

http://creativecommons.org/licenses/by/4.0/

(c) (i) Open Access

\begin{abstract}
This article provides to descript a consistent of the even-even ${ }^{182-200} \mathrm{Pt}$ isotopes. This has been achieved using the interacting boson model-2 (IBM-2) and including configuration mixing (IBM-2 CM). Our attention is paid to describe the nuclei shape and to their connecting with shaping coexistence phenomenon. Ten isotopes are studied, ranging from the middle of the neutron shell to very near the doubly closed shell at ${ }^{208} \mathrm{~Pb}$. The same Hamiltonian is used for all the nuclei studied, with parameters which are constant or smoothly varying. In this study, we showed the transition between more axially symmetric deformed features of light Pt isotopes to $\gamma$-unstable and vibrational isotopes (near spherical shape) for ${ }^{198-200} \mathrm{Pt}$ isotopes.
\end{abstract}

\section{Keywords}

Configuration Mixing, IBM-2, Pt Isotopes, Coexistence Phenomenon

\section{Introduction}

Some nuclei near closed shells appear to have both the vibrational structure expected for a near-spherical shape, and rotational structure, which is typical of deformed nuclei [1]. This phenomenon of shape coexistence involves two configurations of the nucleus which have different numbers of active nucleons. In an IBM description, the two configurations have different boson numbers; $N_{V}$ being the same but $N_{\pi}$ different, or vice versa. The most common situation involves a difference in $N_{\pi}\left(N_{v}\right)$ of two bosons between the normal configuration and the so-called intruder configuration, corresponding to a pair excitation across a shell or sub-shell gap [1] [2].

Shape coexistence in atomic nuclei has become a very active field of research during the last decades and clear signals of its existence have been obtained at and near proton or neutron closed shells [1] [2] [3], more in particular in light 
nuclei with a closed neutron shell at $N=8,20,28$, and 50 closed shells as well as in heavy nuclei such as the $\mathrm{Sn}$ and the $\mathrm{Pb}$ nuclei. It seems that, without exception, shape coexistence is associated with the presence of low-lying excited $0^{+}$states.

In the case of the IBM-2, shape coexistence arises including two-particle two-hole (2p-2h) (or even higher np-nh). Excitations across the closed shells, but they consider them as extra bosons, i.e., pairs of nucleons. This extension is called IBM-2 configuration mixing. In two previous papers [4] [5], we used the IBM-CM to extensively study the Pt nuclei. We carried out a detailed analysis of the energy spectra and absolute $B(E 2)$ values for states up to an energy of 1.5 $\mathrm{MeV}$. Garc'ia-Ramos et al., [5], studied shape evolution and shape coexistence in Pt isotopes: Comparing interacting boson model configuration mixing and Gogny mean-field energy surfaces. In this work, the evolution of the total energy surface and the nuclear shape in the isotopic chain ${ }^{172-194} \mathrm{Pt}$ are studied in the framework of the interacting boson model, including configuration mixing. The results are compared with a self-consistent Hartree-Fock-Bogoliubov calculation using the Gogny-D1S interaction and a good agreement between both approaches is found.

In this article, it will be studied to extract the parameters describing the IBM-2 CM Hamiltonian precisely. After showing some theories and laws about IBM-2, it represents the results of the $\mathrm{Pt}$ isotopes comparing with some experimental data on energy levels, electromagnetic transition probabilities (B(E2) and $\mathrm{B}(\mathrm{M} 1))$, monopole transitions, mixing ratios and quadrupole moments.

\section{The Interacting Boson Model-2 (IBM-2)}

\subsection{The Hamiltonian of Interacting Boson Model-2 (IBM-2)}

As the IBM-2 model, the protons and neutrons degrees of freedom are taken into account explicitly. Consequently, one can write the Hamiltonian [6] [7] as,

$$
\begin{gathered}
H=H_{\pi}+H_{v}+V_{\pi v} \\
H=\varepsilon_{\pi} d_{\pi}^{+} d_{\pi}^{\sim}+\varepsilon_{v} d_{v}^{+} d_{v}^{+}+V_{\pi \pi}+V_{v v}+\kappa Q_{\pi} \cdot Q_{v}+M_{\pi v}
\end{gathered}
$$

Here $\varepsilon$ is the d-boson energy, $\kappa$ is the strength of the quadrupole interacting between proton and neutron bosons.

The quadrupole moment operator, in the IBM-2 model, has the form:

$$
Q_{\rho \rho}=\left(s^{+} d^{\sim}+d^{+} s^{\sim}\right)_{\rho}^{(2)}+\kappa_{\rho}\left(d^{+} d^{\sim}\right)_{\rho}^{(2)}
$$

where $\rho=\pi$ or $v, Q_{\rho \rho}$ is the quadrupole deformation parameter for protons $(\rho=\pi)$ and neutrons $(\rho=v)$. Where the terms $V_{v v}$ and $V_{\pi \pi}$ are the proton-proton and neutron-neutron d-boson interacting only and written by:

$$
V_{\rho \rho}=\sum_{J=0,2,4} \frac{1}{2} C_{L \rho}(2 J+1)^{1 / 2}\left[\left(d^{+} d^{+}\right)_{\rho}^{(2)}(\tilde{d} \tilde{d})_{\rho}^{(2)}\right]^{(0)}
$$

The Majorana interaction is represented the term $M_{\pi v}$, that accounts for the symmetry energy and shits the states with mixed proton-neutron symmetry state with respect to the fully symmetric ones which affects only the relative location 
of the states with mixed symmetry, it is with respect to the totally symmetric states. For the reason that little experimental information is familiar states with mixed symmetry that has the form:

$$
M_{\pi v}=-\sum_{k=1.3} 2 \xi_{k}\left(d_{\pi}^{+} d_{\pi}^{+}\right)^{(k)}\left(d_{\pi}^{\sim} d_{\pi}^{\sim}\right)^{(k)}+\xi_{2}\left(d_{\pi}^{+} s_{v}^{+}-s_{\pi}^{\sim} d_{v}^{\sim}\right)^{(2)}\left(d_{\pi}^{\sim} s_{v}^{\sim}-s_{\pi}^{\sim} d_{v}^{\sim}\right)^{(2)}
$$

\subsection{Electromagnetic Transitions and Quadrupole Moments in IBM-2}

Generally, the E2 transition operator of one-body in the IBM-2 is

$$
\begin{gathered}
T(l)=T_{\pi}(l)+T_{v}(l) \\
T(E 2)=e_{\pi}\left[\left(s^{+} d^{\sim}+d^{+} s^{\sim}\right)_{\pi}^{(2)}+\chi_{\pi}\left(d^{+} d^{\sim}\right)_{\pi}^{(2)}\right]^{(2)} \\
+e_{v}\left[\left(s^{+} d^{\sim}+d^{+} s^{\sim}\right)_{v}^{(2)}+\chi_{v}\left(d^{+} d^{\sim}\right)_{v}^{(2)}\right]^{(2)} \\
T(E 2)=e_{\pi} Q_{\pi}+e_{v} Q_{v}
\end{gathered}
$$

which $Q_{\rho}$ is in the form of Equation (3). For simplicity, the $\chi_{\rho}$ has the same value as in the Hamiltonian. Also, one suggests it by the single $j$-shell microscopy. Generally, the $E 2$ transition results are not sensitive to the choice of $e_{v}$ and $e_{\pi}$, whether $e_{\pi}=e_{v}$ or not. Thus, the reduced electric quadrupole transition rates between $J_{i}^{+} \rightarrow J_{f}^{+}$states are given by:

$$
B\left(E 2 ; J_{i}^{+} \rightarrow J_{f}^{+}\right)=\frac{1}{2 J_{i}+1}\left|\left\langle J_{f}^{+}\|T(E 2)\| J_{i}^{+}\right\rangle\right|^{2}
$$

In IBM-2, the electric quadrupole moment is written as:

$$
Q_{I}=\left[\frac{16 \pi}{5}\right]^{1 / 2}\left[\begin{array}{lll}
J & 2 & J \\
-J & 0 & J
\end{array}\right]\langle J\|T(E 2)\| J\rangle
$$

One can calculate $I B M-2$ eigen functions and energy eigenvalues are usually achieved numerically by the program code $N P B O S$ [8]. Then, the result of eigenvectors can be calculated transition rates and related properties using the program code NPBTRN [8]. The relationship is between the parameters of Equation (2).

\subsection{Configuration Mixing in Interacting Boson Model-2 (IBM-2 CM)}

Configuration mixing can be treated in the IBM-2 using a technique developed by Duval and Barrett [9]. Separate IBM-2 calculations are achieved for the two configurations and the results are then mixed this is done by the interaction configuration mixing can be remedied in the IBM-2 by a technique method developed by Duval and Barrett [9]. Separate IBM-2 calculations are done for the two configurations and the results are then mixed using the interaction

$$
V_{\text {mix }}=\alpha\left(s_{\pi}^{+} s_{\pi}^{+}+s_{\pi} s_{\pi}\right)^{(0)}+\beta\left(d_{\pi}^{+} d_{\pi}^{+}+d_{\pi} d_{\pi}\right)^{(0)}
$$


where the intruder configuration is assumed to involve the proton shell. There are three parameters in the mixing calculation, the mixing strengths $\alpha$ and $\beta$ in Equation (23), and the pair excitation energy, $\Delta$, which gives the relative energies of the two unperturbed configurations.

The total mixing Hamiltonian is then given by

$$
H_{\text {mix }}=H_{1}+H_{2}+V_{\text {mix }}
$$

where $H_{1}\left(H_{2}\right)$ is the IBM-2 Hamiltonian for the first (second) configuration, as given by Equation (11), and an amount $\mathrm{fl}$ has been added to the energies of the second configuration.

The mixed wave functions are used to calculate $B(E 2)$ values of observed transitions and quadrupole moments. The $E 2$ transition operator is given by [9]:

$$
T(E 2)=e_{2}\left[\left(Q_{2 \pi}+Q_{2 v}\right)+\left(e_{4} / e_{2}\right)\left(Q_{4 \pi}+Q_{4 \pi}\right)\right]
$$

where $Q_{\rho}$ were defined in Equation (3) and $e_{j}$ and $e_{\rho j}(j=0,2)$ are adjustable parameters. The suffixes 2 and 4 refer to the normal and the intruder configurations respectively.

For simplicity, $e_{2 \pi}=e_{2 v}=e_{2}$ and $e_{4 \pi}=e_{4 v}=e_{4}$.

For a mathematical simplicity, the neutron boson and proton boson effective charges are often taken to be equal, and the parameters $\chi_{\pi}$ and $\chi_{v}$ (in intruder configuration) are taken to be the same as the Hamiltonian parameters $\chi_{\pi}$ and $\chi_{v}$ respectively. The $T(E 2)$ transition operator can then be written as:

$$
T(E 2)=e_{2}\left(Q_{\pi}+Q_{v}\right)
$$

When two configurations are present, this operator becomes [9]:

$$
T(E 2)=\sum_{i \rho} e_{i \rho} Q_{i \rho}
$$

where $\rho=\pi$ or $v$ and $i$ denotes the configuration. The effective charges of the two configurations are not the same, in general.

\section{Results and Discussion}

\subsection{Choice of Parameters}

The normal configuration for platinum isotopes involves $N_{\pi}=2$ (sometimes denoted as $2 \pi$, two proton boson holes), counting from the $Z=82$ closed shell. The neutron configuration for ${ }_{78}^{196} \mathrm{Pt}_{118}$ for example, is $N_{v}=2$ (four neutron boson holes), counting from the $N=126$ closed shell. The vibrational spectra can be calculated by diagonalizing the IBM-2 Hamiltonian, (Equation (2)), in the space of two proton and $N_{v}$ neutron $s$ and $d$ bosons. In order to describe the rotational states, an alternative configuration must be specified and a separate set of IBM-2 calculations made, based on that configuration. The alternate configuration used for the ${ }^{186-200} \mathrm{Pt}$ isotopes involves a two-particle-four-hole excitation in the shell model proton space [1] [9] [10]. This corresponds to two proton boson particles and two proton boson hole in the IBM-2 space. For simplicity, the proton boson particles and hole are treated equivalently, even though 
the underlying fermion pair degrees of freedom originate in different major shells.

The IBM-2 calculations have been done in model spaces with $N_{\pi}=2(2 \pi)$ and $N_{\pi}=4(4 \pi)$ to describe the vibrational and rotational states, respectively, the two calculations are combined using Equation (11).

In the phenomenological calculations the parameters appearing in the Hamiltonian ((Equation (2) and Equation (11)) in two configurations may in general depend both on proton $\left(N_{\pi}\right)$ and neutron $\left(N_{v}\right)$ boson number. Guided by the microscopic theory as we have assumed that only $\varepsilon^{2 \pi}, \varepsilon^{4 \pi}$ and $\kappa^{2 \pi}, \kappa^{4 \pi}$ depend on $N_{\pi}(2 \pi, 4 \pi)$ and $N_{v}$ i.e., $\varepsilon\left(N_{\pi}, N_{v}\right), \kappa\left(N_{\pi}, N_{v}\right)$, while $\chi_{v}$ depends only on $N_{v}$ and $\chi_{\pi}$ on $N_{\pi}$, i.e., $\chi_{v}\left(N_{v}\right), \chi_{\pi}\left(N_{\pi}\right)$. Thus a set of isotopes, (constant $N_{v}$ ) have the same value of $\chi_{v}$, while a set of isotones, (constant $N_{\pi}$ ), have the same value of $\chi_{\pi}$. This parameterization allows one to correlate a large number of experimental data. Similarly, when a proton-proton $V_{\pi \pi}$ and neutron-neutron $V_{v V}$, interaction is added, the coefficients $C_{L}$ are taken as $C_{L \pi}\left(N_{\pi}\right)$ and $C_{L v}\left(N_{v}\right)$, i.e. the proton boson interaction will only depend on $N_{\pi}$, and the neutron boson on $N_{v}$. Since there is no information on the location of the states with mixed neutron-proton symmetry we kept the coefficients appearing in the Majorana force $M_{\pi v}$ (see Table 1 and Table 2).

The values of the parameters used for the present calculations are given in Table 1. The value of the parameter (boson energy) $\varepsilon$ for the $N_{\pi}=4$ configuration, $\varepsilon^{4 \pi}$, is constant for all isotopes and the values of $\varepsilon$ for the $N_{\pi}=2$ configuration, $\varepsilon^{2 \pi}$, are nearly constant. The quadrupole-quadrupole interaction strengths $\kappa$ trends for $(2 \pi)$ both configurations follow the microscopic predictions [9].

The values of the parameter $\chi_{v}$ used for Pt isotopes are the microscopic predictions from Bijker et al., 1980 [11]. They were reported only for neutron number $102 \leq N \leq 122$. The values of $\chi_{v}$ for Pt isotopes were determined by extrapolating the microscopic trend to larger neutron number. This was done in

Table 1. IBM-2 Hamiltonian parameters for the Pt isotopes (normal configuration $N_{\pi}=2$ ). All energies are in $\mathrm{MeV} ; \chi_{\pi}^{2 \pi}$ and $\chi_{v}^{2 \pi}$ are dimensionless.

\begin{tabular}{cccccccc}
\hline Nuclei & $\varepsilon^{2 \pi}$ & $\kappa^{2 \pi}$ & $\chi_{v}^{2 \pi}$ & $\chi_{\pi}^{2 \pi}$ & $C_{0 v}^{2 \pi}$ & $C_{2 v}^{2 \pi}$ & $C_{4 v}^{2 v}$ \\
\hline${ }^{186} \mathrm{Pt}$ & 0.610 & -0.148 & -0.52 & -0.810 & -0.250 & -0.160 & 0.00 \\
${ }^{188} \mathrm{Pt}$ & 0.580 & -0.161 & 0.00 & -0.810 & -0.250 & -0.160 & 0.00 \\
${ }^{190} \mathrm{Pt}$ & 0.580 & -0.178 & 0.46 & -0.810 & 0.00 & -0.127 & 0.00 \\
${ }^{192} \mathrm{Pt}$ & 0.580 & -0.180 & 0.81 & -0.810 & 0.450 & -0.09 & 0.00 \\
${ }^{1946} \mathrm{Pt}$ & 0.580 & -0.180 & 0.95 & -0.810 & 0.500 & 0.00 & 0.00 \\
${ }^{196} \mathrm{Pt}$ & 0.580 & -0.180 & 1.05 & -0.810 & 0.560 & 0.002 & 0.00 \\
${ }^{198} \mathrm{Pt}$ & 0.580 & -0.180 & 1.07 & -0.810 & 0.750 & 0.0018 & 0.00 \\
${ }^{200} \mathrm{Pt}$ & 0.590 & -0.180 & 1.08 & -0.810 & 0.800 & 0.001 & 0.00 \\
\hline
\end{tabular}

$\xi_{1}=\xi_{3}=0.11, \quad \xi_{2}=0.0, \quad C_{0 \pi}^{2 \pi}=C_{2 \pi}^{2 \pi}=C_{4 \pi}^{2 \pi}=0.00$. 
Table 2. IBM-2 Hamiltonian parameters for the Pt isotopes (normal configuration $\left.N_{\pi}=4\right)$. (2P-2h), All energies are in $\mathrm{MeV} ; \quad \chi_{\pi}^{4 \pi}$ and $\chi_{v}^{4 \pi} \quad \chi_{v}^{2 \pi}$ are dimensionless.

\begin{tabular}{cccccccc}
\hline Nuclei & $\varepsilon^{4 \pi}$ & $\kappa^{4 \pi}$ & $\chi_{v}^{4 \pi}$ & $\chi_{\pi}^{4 \pi}$ & $C_{0 v}^{4 \pi}$ & $C_{2 v}^{4 \pi}$ & $C_{4 v}^{4 \pi}$ \\
\hline${ }^{186} \mathrm{Pt}$ & 0.550 & -0.155 & 1.080 & -1.10 & -0.250 & -0.160 & 0.00 \\
${ }^{188} \mathrm{Pt}$ & 0.550 & -0.150 & 1.070 & -1.10 & -0.250 & -0.160 & 0.00 \\
${ }^{190} \mathrm{Pt}$ & 0.550 & -0.145 & 1.050 & -1.10 & 0.00 & -0.127 & 0.00 \\
${ }^{192} \mathrm{Pt}$ & 0.550 & -0.140 & 0.950 & -1.10 & 0.450 & -0.09 & 0.00 \\
${ }^{1946} \mathrm{Pt}$ & 0.550 & -0.135 & 0.811 & -1.10 & 0.500 & 0.00 & 0.00 \\
${ }^{196} \mathrm{Pt}$ & 0.550 & -0.130 & 0.461 & -1.10 & 0.560 & 0.002 & 0.00 \\
${ }^{198} \mathrm{Pt}$ & 0.550 & -0.125 & 0.00 & -1.10 & 0.750 & 0.0018 & 0.00 \\
${ }^{200} \mathrm{Pt}$ & 0.550 & -0.120 & -0.520 & -1.10 & 0.800 & 0.001 & 0.00 \\
\hline
\end{tabular}

$\xi_{1}=\xi_{3}=-0.11, \quad \xi_{2}=0.0, \quad C_{0 \pi}^{4 \pi}=C_{2 \pi}^{4 \pi}=C_{4 \pi}^{4 \pi}=0.00$.

a manner consistent with the phenomenologically determined $\chi_{v}$ values which were used for the neighboring platinum and osmium isotones [11]. The same values of $\chi_{v}$ are used for both configurations.

The emphasis in this work is on describing overall trends with constant or smoothly varying parameters of the Hamiltonian (Equations. (2), (11)), rather than obtaining the best possible fit to the experimental data for each nucleus. This is done in an effort to find a set of IBM-2Hamiltonian parameters which is appropriate for the entire isotopic chain.

The normal configuration for platinum involves $N_{\pi}=2$ (sometimes $(2 \pi)$ denoted as ( $2 \pi)$, two proton boson hole), counting from the $Z=82$ closed shell. The neutron configuration for ${ }^{196} \mathrm{Pt}$ for example, is $N_{v}^{-}=4$ (four neutron boson holes), counting from the $N=126$ closed shell. The vibrational spectra can be calculated by diagonalizing the IBM-2 Hamiltonian, Equation (2), in the space of one proton and $N_{v}=4$ neutron $s$ and $d$ bosons. In order to describe the rotational states, an alternative configuration must be specified and a separate set of IBM-2 calculations made, based on that configuration. The alternate configuration used for the Pt isotopes involves a four-particle-four-hole excitation in the shell model proton space. This corresponds to two proton boson particles and two proton boson hole in the IBM-2 space. The two configurations are depicted schematically energy levels in Table 2 . For simplicity, the proton boson particles and hole are treated equivalently, even though the underlying fermion pair degrees of freedom originate in different major shells.

\subsection{Energy Spectra}

The configuration mixing calculations are done using the computer code NPMIX [10], which calculates the energy eigenvalues and eigenfunctions. The computer codes NPBEMX and BEMIX [10] are subsequently used to calculate matrix elements for transition rates and other properties.

The mixing between the two configurations $(2 \pi)$ and $(4 \pi)$ is apparent in the 
experimental data shown in Figures 1-3. Consider, for example, the $4_{1}^{+}, 0_{2}^{+}$ and $4_{2}^{+}, 0_{2}^{+}$states in ${ }^{186-196} \mathrm{Pt}$ isotopes. In the latter, the vibrational (2) state is lower in energy than the rotational $(4 \pi) 4_{1}^{+}$and $4_{2}^{+}$states. The states are close in energy and mutually repel, with the vibrational $4^{+}$state being lower in energy and the rotational $4^{+}$state higher in energy than would be expected without configuration mixing.

In the second configuration $(4 \pi)$ configuration interaction parameters $C_{2 v}^{2 \pi}=C_{2 v}^{4 \pi}, \quad C_{0 v}^{2 \pi}=C_{0 v}^{4 \pi}=0$ and $C_{4 v}^{2 \pi}=C_{4 v}^{4 \pi}=0$ for all isotopes. For most nuclei, the spectral structure comes primarily from the pairing and quadrupole-quadrupole terms in the IBM-2 Hamiltonian, Equation (2), and the $V_{v v}$ term is relatively unimportant. Since there is only two proton boson to interact through Q.Q, the residual interaction among the neutron bosons $V_{v v}$ interaction is comparatively large.

From Figure 1, in the normal configuration the ground state band the moment of inertia increases, beta band $\left(0_{2}^{+}, 2_{2}^{+}, 4_{2}^{+}\right)$is pushed up, where the state $0_{2}^{+}$becomes bandhead (a member of $\gamma$-band) of $K^{\pi}=0^{+}$. In ${ }^{194-200} \mathrm{Pt}$ isotopes, only five $0_{2}^{+}$states are observed around $1.25 \mathrm{MeV}$ is produced, some of these states do not have a $S U(6)_{\pi} \otimes S U(6)_{v}$ symmetry.

In the IBM-2-CM approach, the lightest Pt isotopes, are deformed $\left({ }^{172-178} \mathrm{Pt}\right)$. In ${ }^{186} \mathrm{Pt}$ isotope a prolate shape and a $\gamma$-soft minimum coexist, but a well-deformed prolate minimum quickly develops in ${ }^{180} \mathrm{Pt}$ isotope, becoming the most pronounced prolate minimum at the mid-shell, i.e., in ${ }^{182} \mathrm{Pt}$ isotope with the prolate shape remaining well pronounced up to ${ }^{186} \mathrm{Pt}$ isotope. Moving towards heavier mass $\mathrm{Pt}$ isotopes, $\gamma$-flat energy surfaces start to develop. For ${ }^{188} \mathrm{Pt}$ isotope, a much extended energy surface develops in the $\gamma$-direction (deviation from symmetry axis), becoming completely $\gamma$-unstable when reaching ${ }^{190-196} \mathrm{Pt}$ isotopes, the ${ }^{198-200} \mathrm{Pt}$ isotopes tends to spherical shape because approach to magic number.

We show in Figure 1 the evolution of the energy of the ground state band $\left(2_{1}^{+}\right.$, $4_{1}^{+}, 6_{1}^{+}$, and $8_{1}^{+}$) increases toward the middle of the major shell with the number of the valence neutrons and remain almost constant for $180 \leq A \leq 186$ isotopes. Although these tendencies are well reproduced, the rotational properties or rotational features are somewhat enhanced in the calculated levels for $180 \leq A \leq 184$, which are slightly agreement in energy with the experimental data. From both results, the IBM-2 CM and the experimental data, we observed the fingerprints for structural evolution with a jump between ${ }^{186} \mathrm{Pt}$ and ${ }^{188} \mathrm{Pt}$ isotopes, also the shape of the nucleus changed from prolate ( ${ }^{186} \mathrm{Pt}$ isotope) to oblate $\left({ }^{188} \mathrm{Pt}\right.$ isotope) deformation, this case is called phase transition. The ${ }^{188} \mathrm{Pt}$ isotope takes the oblate shape because the yrast states gradually departure to neutron closed shell.

One can also find signatures for a shape or phase transition in the systematics of the quasi- $\beta$-band levels shown in Figure 2 . In ${ }^{180-186} \mathrm{Pt}$ isotopes configuration mixing, the $0_{2}^{+}$baldhead and the state $2_{3}^{+}$nearly constant in both IBM-2 data and experimental data. The two levels are pushed up rather significantly from 


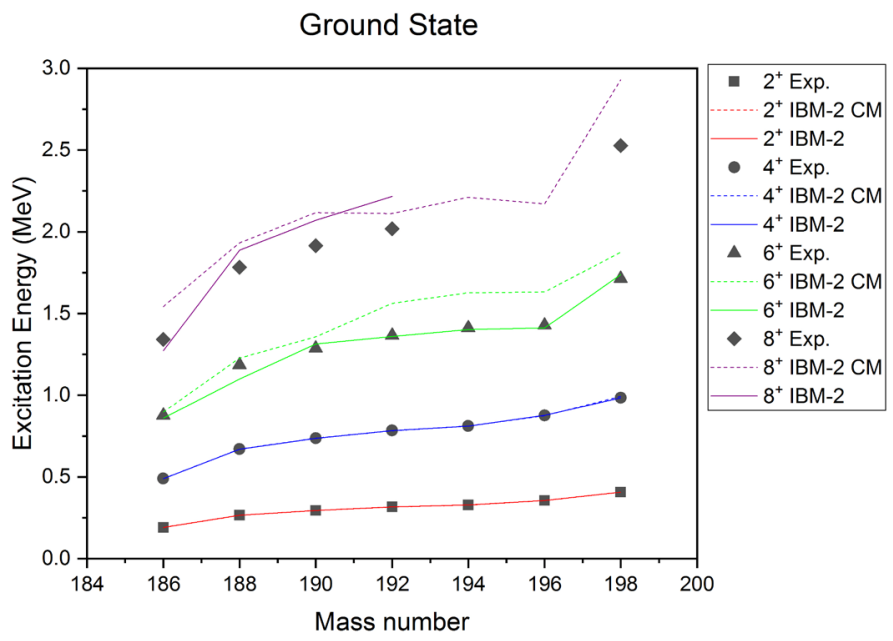

Figure 1. Ground state band in Pt isotopes. Experimental data are taken from Refs. [12]-[21].

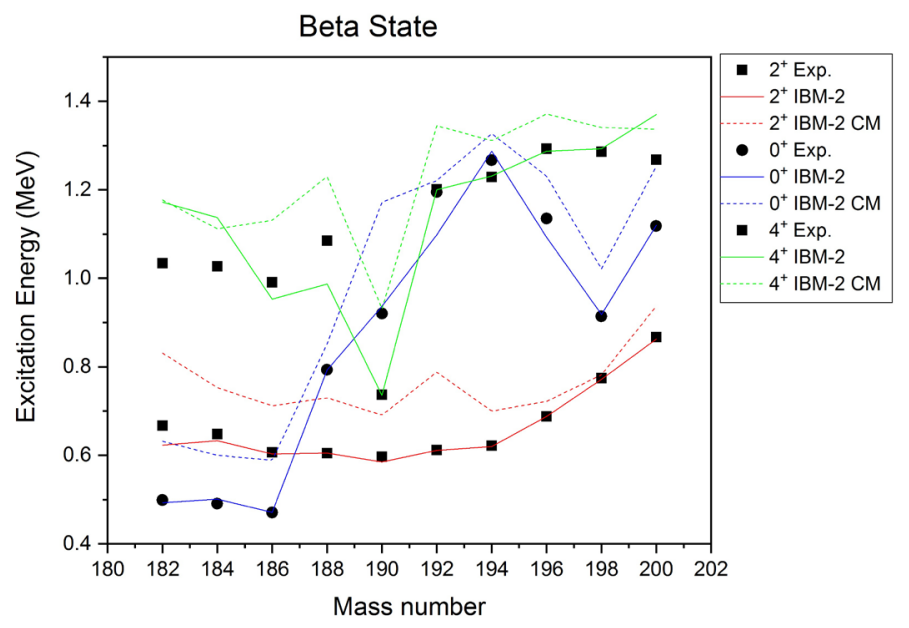

Figure 2. Beta state band in Pt isotopes. Experimental data are taken from Refs. [12]-[21].

${ }^{186} \mathrm{Pt}$ isotope to ${ }^{188} \mathrm{Pt}$ isotope. Consistently with the systematics in the ground-state band. The calculated $0_{2}^{+}$and $2_{3}^{+}$states are higher than, but still follow, the experimental data. The $3_{1}^{+}$state lies close to the $4_{2}^{+}$state. However, this calculation predicts that this trend persists even for ${ }^{188-198} \mathrm{Pt}$ isotopes, whereas the energy spacing between the experimental $3_{1}^{+}$and $4_{2}^{+}$states for these isotopes is larger. Similar deviation occurs for high spin states. This means that our calculations suggest the feature characteristic of the $\mathrm{O}(6)$ symmetry ( $\gamma$-unstable).

The $\gamma$-band states shown in Figure 3, we can see the good agreement between IBM-2 calculations and experimental data for $180 \leq A \leq 188$, where the $3_{1}^{+}$, $4_{3}^{+}$and $2_{3}^{+}$states are energetically closed.

The high spin states for example., $6_{2}^{+}, 3_{2}^{+}, 4_{4}^{+}$, states, we observe some deviation occurs for these states. This means that our calculations suggest the feature characteristic of the $\mathrm{O}(6)$ symmetry, where the staggering occurs as high spin beta band states such as $2_{3}^{+}, 4_{3}^{+}, 6_{2}^{+}, \ldots$, etc., However, the experimental 


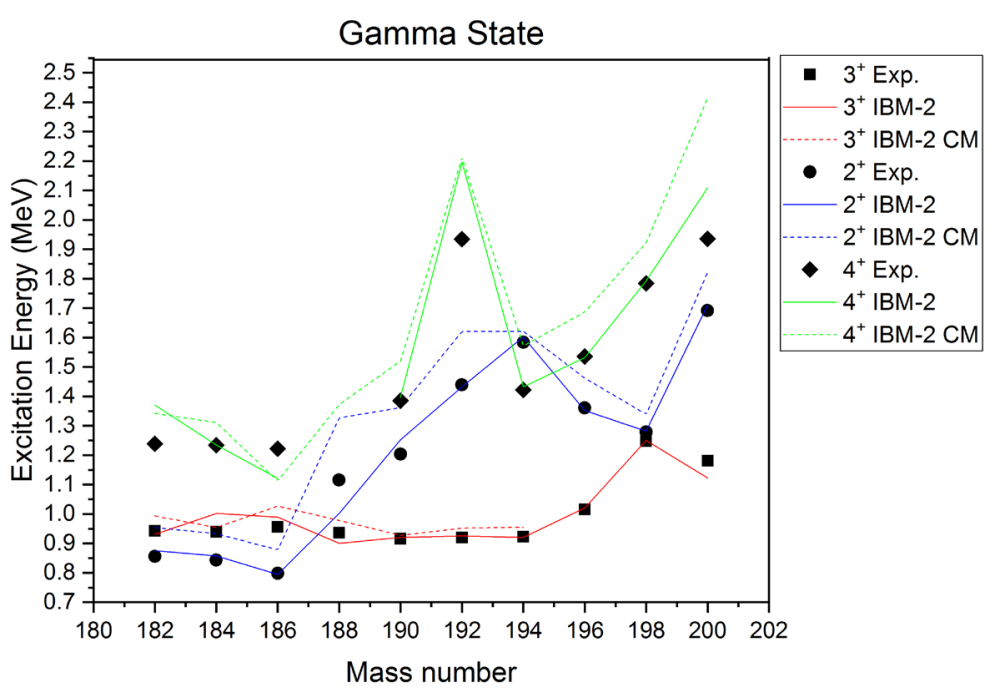

Figure 3. Gamma state band in Pt isotopes. Experimental data are taken from Refs. [12]-[21].

levels are lying more regularly, particularly for $188 \leq A \leq 196$, and thus appear to be in between the $\mathrm{O}(6)$ symmetry and triaxial rotor symmetry. The deviation of the $\gamma$-band structure seems to be nothing more than a consequence of an algebraic nature of the IBM-2, and indeed has also been found in existing phenomenologically calculations of IBM-2.

A characteristic feature of the $\gamma$-unstable limit of the IBM-2 is a bunching of $\gamma$-band states according to $2^{+},\left(3^{+}, 4^{+}\right),\left(5^{+}, 6^{+}\right), \ldots$, that is, $3^{+}$and $4^{+}$are close in energy, etc. This even-even staggering is observed in certain $\mathrm{O}(6)$ nuclei but not in all and in some it is, in fact, replaced by the opposite bunching $\left(2^{+}, 3^{+}\right),\left(4^{+}\right.$, $\left.5^{+}\right), \ldots$ which is typical of a rigid triaxial rotor [22]. From these qualitative observations it is clear that the even-even $\gamma$-band staggering is governed by the $\gamma$-degree of freedom (i.e., triaxiality) as it changes character in the transition from a $\gamma$-soft vibrator to a rigid triaxial rotor. The energy surface becomes rather flat, evolving towards a spherical minimum at ${ }^{200} \mathrm{Pt}$ and beyond. The possibility of triaxial deformation was not considered (tends to spherical shape).

\subsection{Electric Transition Probability B(E2)}

Calculations of electromagnetic properties give us a good test of the nuclear models prediction. The electromagnetic matrix elements between eigenstates were calculated using the programs NPBTRN and NPBEMX for IBM-2 and IBM-2 CM model respectively.

From Equations (7), we note that an E2 transition mainly depends on identifying proton and neutron bosons effective charges $e_{\pi}$ and $e_{v}$. These isotopes lying in region between $\mathrm{SU}(3)$ limit (rotational nuclei) and $\mathrm{O}(6)$ limit ( $\gamma$-soft nuclei), therefore, the relationship between $\left(e_{\pi}, e_{v}\right)$ and the reduced transition probability $\mathrm{B}(\mathrm{E} 2)$ for rotational limit $\mathrm{SU}(3)$ and $\gamma$-soft limit $\mathrm{O}(6)$ is given in the form [23] [24]: 
For $\mathrm{O}(6)$ limit

$$
B\left(E 2 ; 2_{1}^{+} \rightarrow 0_{1}^{+}\right)=\frac{(N+4)\left(e_{\pi} N_{\pi}+e_{v} N_{v}\right)}{5 N}
$$

For SU(3) limit

$$
B\left(E 2 ; 2_{1}^{+} \rightarrow 0_{1}^{+}\right)=\frac{(2 N+3)\left(e_{\pi} N_{\pi}+e_{v} N_{v}\right)}{5 N}
$$

where $B\left(E 2 ; 2_{1}^{+} \rightarrow 0_{1}^{+}\right)$is the experimental reduced transition probability from the first excited states $\left(2_{1}^{+}\right)$to the ground state $\left(0_{1}^{+}\right), N$ is the total number of bosons.

The relations (26) and (27), was used to estimate the effective boson charges for proton and neutron bosons $\left(e_{\pi}, e_{v}\right)$. In these calculations, we use the following criteria to determine the effective charges. $e_{\pi}=0.173$ e.b is a constant throughout the whole isotopic chain and the $e_{v}$ changes with neutron number. This is true if the neutron (proton) interaction does not depend on the proton (neutron) configurations. The values of $e_{\pi}$ and $e_{v}$ are determined by fitting to the five $B\left(E 2 ; 2_{1}^{+} \rightarrow 0_{1}^{+}\right)$and $B\left(E 2 ; 2_{2}^{+} \rightarrow 2_{1}^{+}\right)$in ${ }^{194} \mathrm{Pt}$ and ${ }^{196} \mathrm{Pt}$ isotopes from the first configuration $\left(N_{\pi}=2\right)$. They are given in Table 3 .

For the configuration mixing, the effective charges for bosons is evaluated in the same manner in normal configuration, For simplicity, $e_{2 \pi}=e_{2 v}=e_{2}$ and $e_{4 \pi}=e_{4 v}=e_{4}$. The ratio of the two quadrupole effective charges, $e_{4} / e_{2}$ in Equation (25) is taken to be the same quadrupole interaction strengths, as the ratio of the corresponding $\kappa^{4 \pi} / \kappa^{2 \pi}$, for each isotope (see Equation (12) and Table 1 , Table 2). This is reasonable, since the effective charge and the strength of the quadrupole interaction are both proportional to the mean square proton radius. Thus, the only new parameter needed to determine the reduced transition rates is $e_{2}$. The value for the $\mathrm{Pt}$ isotopes was determined by fitting of $B\left(E 2 ; 2_{1}^{+} \rightarrow 0_{1}^{+}\right)$in ${ }^{196} \mathrm{Pt}$ isotope.

It is well known that absolute gamma ray transition probabilities offer the possibility of a very sensitive test of nuclear models and the majority of the information on the nature of the ground state has come from studies of the energy level spacing. The transition probability values of the excited state in the ground state band constitute another source of nuclear information. Yrast levels of even-even nuclei $\left(\mathrm{J}^{\pi}=2^{+}, 4^{+}, 6^{+}, \cdots\right)$ usually decay by $E 2$ transition to the lower lying yrast level with $J_{f}^{+}=J_{i}^{+}-2$.

In Table 4 we show the $B\left(E 2 ; 2_{1}^{+} \rightarrow 0_{1}^{+}\right)$and $B\left(E 2 ; 4_{1}^{+} \rightarrow 2_{1}^{+}\right)$values, which are of the same order of magnitude and display a typical decrease towards the middle of the shell.

As a consequence of possible $M 1$ admixture the $B\left(E 2 ; 3_{1}^{+} \rightarrow 2_{1}^{+}\right)$quantity is rather difficult to measure. There is no experimental data to compare the values

\begin{tabular}{|c|c|c|c|c|c|c|c|c|c|c|c|}
\hline Isotopes & ${ }^{180} \mathrm{Pt}$ & ${ }^{182} \mathrm{Pt}$ & ${ }^{184} \mathrm{Pt}$ & ${ }^{186} \mathrm{Pt}$ & ${ }^{188} \mathrm{Pt}$ & ${ }^{190} \mathrm{Pt}$ & ${ }^{192} \mathrm{Pt}$ & ${ }^{194} \mathrm{Pt}$ & ${ }^{196} \mathrm{Pt}$ & ${ }^{198} \mathrm{Pt}$ & ${ }^{200} \mathrm{Pt}$ \\
\hline $\begin{array}{ll}e_{v} & (\mathrm{eb})\end{array}$ & 0.1243 & 0.126 & 0.1286 & 0.128 & 0.129 & 0.1295 & 0.132 & 0.138 & 0.142 & 0.143 & 0.144 \\
\hline
\end{tabular}

Table 3. Effective charge used in $\mathrm{E} 2$ transition calculations ( $\left.e_{\pi}=0.173 \mathrm{e.b}\right)$. 
Table4. Electric transition probability $B\left(E 2 ; J_{i}^{+} \rightarrow J_{f}^{+}\right)$in $\mathrm{e}^{2} \mathrm{~b}^{2}$ units.

\begin{tabular}{|c|c|c|c|c|}
\hline Nuclei & $J_{i}^{+} \rightarrow J_{f}^{+}$ & Exp. & IBM-2 & IBM-2 CM \\
\hline \multirow{4}{*}{ Pt- 186} & $2_{1}^{+} \rightarrow 0_{1}^{+}$ & $0.59[14]$ & 0.572 & 0.583 \\
\hline & $2_{2}^{+} \rightarrow 0_{1}^{+}$ & - & 0.032 & 0.0431 \\
\hline & $2_{2}^{+} \rightarrow 2_{1}^{+}$ & - & 0.112 & 0.143 \\
\hline & $4_{1}^{+} \rightarrow 2_{1}^{+}$ & - & 0.140 & 0.173 \\
\hline \multirow{4}{*}{ Pt- 188} & $2_{1}^{+} \rightarrow 0_{1}^{+}$ & $0.520(94)[25]$ & 0.522 & 0.522 \\
\hline & $2_{2}^{+} \rightarrow 0_{1}^{+}$ & - & 0.0021 & 0.027 \\
\hline & $2_{2}^{+} \rightarrow 2_{1}^{+}$ & - & 0.698 & 0.0963 \\
\hline & $4_{1}^{+} \rightarrow 2_{1}^{+}$ & - & 0.721 & 0.822 \\
\hline \multirow{5}{*}{ Pt- 190} & $2_{1}^{+} \rightarrow 0_{1}^{+}$ & $0.350(44)[25]$ & 0.350 & 0.355 \\
\hline & $2_{2}^{+} \rightarrow 0_{1}^{+}$ & - & 0.018 & 0.021 \\
\hline & $2_{2}^{+} \rightarrow 2_{1}^{+}$ & - & 0.440 & 0.472 \\
\hline & $4_{1}^{+} \rightarrow 2_{1}^{+}$ & - & 0.634 & 0.827 \\
\hline & $3_{1}^{+} \rightarrow 2_{1}^{+}$ & $1.800(13)[26]$ & 1.972 & 1.873 \\
\hline \multirow{5}{*}{ Pt- 192} & $2_{1}^{+} \rightarrow 0_{1}^{+}$ & $0.382(12)[27]$ & 0.382 & 0.389 \\
\hline & $2_{2}^{+} \rightarrow 0_{1}^{+}$ & $0.0044(5)[27]$ & 0.006 & 0.0072 \\
\hline & $2_{2}^{+} \rightarrow 2_{1}^{+}$ & $0.460(5)[27]$ & 0.481 & 0.487 \\
\hline & $4_{1}^{+} \rightarrow 2_{1}^{+}$ & $0.580(30)[27]$ & 0.597 & 0.544 \\
\hline & $3_{1}^{+} \rightarrow 2_{1}^{+}$ & $0760(15)[25]$ & 0.852 & 0.776 \\
\hline \multirow{10}{*}{ Pt- 194} & $2_{1}^{+} \rightarrow 0_{1}^{+}$ & $0.324(16)[28]$ & 0.324 & 0.327 \\
\hline & $4_{1}^{+} \rightarrow 2_{1}^{+}$ & $0.470(3)[28]$ & 0.476 & 0.467 \\
\hline & $6_{1}^{+} \rightarrow 4_{1}^{+}$ & $0.320(8)[28]$ & 0.331 & 0.351 \\
\hline & $8_{1}^{+} \rightarrow 6_{1}^{+}$ & $0.360(11)[28]$ & 0.421 & 0.339 \\
\hline & $4_{2}^{+} \rightarrow 4_{2}^{+}$ & $0.280(12)[28]$ & 0.310 & 0.263 \\
\hline & $6_{2}^{+} \rightarrow 4_{2}^{+}$ & $0.280(12)[28]$ & 0.279 & 0.331 \\
\hline & $2_{2}^{+} \rightarrow 0_{1}^{+}$ & $0.0014(2)[28]$ & 0.0019 & 0.0175 \\
\hline & $2_{2}^{+} \rightarrow 2_{1}^{+}$ & $0.423(15)[29]$ & 0.520 & 0.631 \\
\hline & $4_{2}^{+} \rightarrow 4_{1}^{+}$ & $0.870(43)[29]$ & 0.887 & 0.883 \\
\hline & $4_{2}^{+} \rightarrow 2_{1}^{+}$ & $0.01(5)[29]$ & 0.027 & 0.0289 \\
\hline \multirow{11}{*}{ Pt- 196} & $2_{1}^{+} \rightarrow 0_{1}^{+}$ & $0.280(8)[25]$ & 0.280 & 0.281 \\
\hline & $2_{2}^{+} \rightarrow 0_{1}^{+}$ & $0.318(23)[11]$ & 0.320 & 0.325 \\
\hline & $4_{1}^{+} \rightarrow 2_{1}^{+}$ & $0.409(22)[11]$ & 0.412 & 0.411 \\
\hline & $4_{2}^{+} \rightarrow 2_{1}^{+}$ & $0.003(1)[30]$ & 0.006 & 0.0081 \\
\hline & $4_{2}^{+} \rightarrow 4_{1}^{+}$ & $0.139(97)[11]$ & 0.192 & 0.187 \\
\hline & $0_{2}^{+} \rightarrow 2_{2}^{+}$ & $0.142(77)[25]$ & 0.144 & 0.146 \\
\hline & $0_{2}^{+} \rightarrow 2_{1}^{+}$ & $0.022(10)[11]$ & 0.023 & 0.042 \\
\hline & $4_{2}^{+} \rightarrow 2_{2}^{+}$ & $0.177(25)[30]$ & 0.182 & 0.207 \\
\hline & $6_{1}^{+} \rightarrow 4_{1}^{+}$ & $0.421(116)[30]$ & 0.427 & 0.521 \\
\hline & $2_{2}^{+} \rightarrow 0_{1}^{+}$ & $0.318(23)[30]$ & 0.337 & 0.355 \\
\hline & $3_{1}^{+} \rightarrow 2_{1}^{+}$ & $0.130(11)[30]$ & 0.136 & 0.147 \\
\hline
\end{tabular}




\begin{tabular}{ccccc}
\hline & $2_{1}^{+} \rightarrow 0_{1}^{+}$ & $0.212(10)[25]$ & 0.212 & 0.200 \\
& $2_{2}^{+} \rightarrow 0_{1}^{+}$ & $0.0003(1)[30]$ & 0.00076 & 0.0005 \\
& $2_{2}^{+} \rightarrow 2_{1}^{+}$ & $0.262(38)[30]$ & 0.289 & 0.311 \\
& $4_{1}^{+} \rightarrow 2_{1}^{+}$ & $0.2700(23)[30]$ & 0.297 & 0.297 \\
& $2_{1}^{+} \rightarrow 0_{1}^{+}$ & - & 0.200 & 0.202 \\
& $2_{2}^{+} \rightarrow 0_{1}^{+}$ & - & 0.00089 & 0.0008 \\
& $2_{2}^{+} \rightarrow 2_{1}^{+}$ & - & 0.275 & 0.245 \\
& $4_{1}^{+} \rightarrow 2_{1}^{+}$ & - & 0.281 & 0.310 \\
\hline
\end{tabular}

of IBM-2. For ${ }^{190} \mathrm{Pt}$ isotope, we give the agreement with experimental value, from these values seems to decrease for ${ }^{190-196} \mathrm{Pt}$ isotopes.

In Table 4, we show $B\left(E 2 ; 2_{2}^{+} \rightarrow 0_{1}^{+}\right)$values. Experimentally the results are radically different for the Ptisotopes. In some Ptisotopes the value seems to decreased towards the middle of the shell, whereas in another ${ }^{196} \mathrm{Pt}$ isotope is increased. Our calculations could not reproduce these contradictory features simultaneously. The results for $B\left(E 2 ; 2_{2}^{+} \rightarrow 0_{1}^{+}\right)$values are shown in Table 4 . This quantity is rather small since this transition is forbidden in all three symmetries of the IBM-2.

As a consequence of possible $M 1$ admixture the $B\left(E 2 ; 2_{2}^{+} \rightarrow 2_{1}^{+}\right)$quantity is rather difficult to measure. For some Ptisotopes, we give the different, conflicting experimental results and we see that no general feature be derived from them, from these values seems to decrease for Ptisotopes.

In ${ }^{194} \mathrm{Pt}$ isotope, there is a good agreement between experimental and calculated $B\left(E 2 ; 0_{2}^{+} \rightarrow 2_{1}^{+}\right)$value. This confirms our earlier statement about the nature of the lowest $O_{2}^{+}$state in this isotope. Other transitions such as $B\left(E 2 ; 6_{1}^{+} \rightarrow 4_{1}^{+}\right), \quad B\left(E 2 ; 8_{1}^{+} \rightarrow 6_{1}^{+}\right)$and $B\left(E 2 ; 4_{2}^{+} \rightarrow 2_{2}^{+}\right)$are small values in sometimes because these transitions between different bands (cross over transitions) and the selection rules which determine these transition.

In Table 5, the quadrupol moment, qualitatively, for first excited state $Q\left(2_{1}^{+}\right)$, second excited states $Q\left(2_{2}^{+}\right)$and $Q\left(4_{1}^{+}\right)$, for the ground state band, the positive $Q\left(2_{1}^{+}\right)$and $Q\left(4_{1}^{+}\right)$mean a negative intrinsic quadrupole moment (for ground state $Q_{0}$ ). For the beta band, a negative $Q\left(2_{1}^{+}\right)$means a negative intrinsic quadrupole moment $Q_{0}$. The negative $Q_{0}$ implies that the nucleus has an oblate shape; the positive intrinsic quadrupole moment $Q_{0}$ means that the nucleus has a prolate shape. The overall the IBM-2 and IBM-2 $\mathrm{CM}$ results is a good agreement with the experimental data.

\section{Concluding Remarks}

In the present work, we have analyzed the spectroscopic properties for the $\mathrm{Pt}$ isotopic chain ${ }^{182-200} \mathrm{Pt}$ in terms of the interacting boson model-2 (IBM-2) and interacting boson model configuration mixing (IBM-2 CM). Calculations of energy levels for even-even ${ }^{182-200} \mathrm{Pt}$ isotopes were performed with the whole 
Table 5. Quadrupole moments $Q\left(J_{i}^{+}\right)$in eb Units for Pt isotopes.

\begin{tabular}{|c|c|c|c|c|}
\hline Nuclei & $J_{i}^{+}$ & Exp. & IBM-2 & IBM-2 CM \\
\hline & $2_{1}^{+}$ & - & 0.096 & 0.091 \\
\hline \multirow[t]{3}{*}{ Pt-186 } & $2_{2}^{+}$ & - & -0.082 & -0.065 \\
\hline & $4_{1}^{+}$ & - & 0.088 & 0.079 \\
\hline & $2_{1}^{+}$ & - & 0.081 & 0.087 \\
\hline \multirow[t]{3}{*}{ Pt-188 } & $2_{2}^{+}$ & - & -0.072 & -0.079 \\
\hline & $4_{1}^{+}$ & - & 0.182 & 0.188 \\
\hline & $2_{1}^{+}$ & - & 0.556 & 0.621 \\
\hline \multirow[t]{3}{*}{ Pt-190 } & $2_{2}^{+}$ & - & -0.522 & -0.529 \\
\hline & $4_{1}^{+}$ & - & 0.571 & 0.627 \\
\hline & $2_{1}^{+}$ & $0.55(21)[17]$ & 0.53 & 0.580 \\
\hline \multirow[t]{3}{*}{ Pt-192 } & $2_{2}^{+}$ & - & -0.62 & -0.68 \\
\hline & $4_{1}^{+}$ & - & 0.58 & 0.61 \\
\hline & $2_{1}^{+}$ & [18] $0.63(6)$ & 0.650 & 0.697 \\
\hline \multirow[t]{3}{*}{ Pt-194 } & $2_{2}^{+}$ & [18] $-0.5(5)$ & -0.52 & -0.62 \\
\hline & $4_{1}^{+}$ & [18] $0.5(10)$ & 0.503 & 0.58 \\
\hline & $2_{1}^{+}$ & [19] $0.62(8)$ & 0.64 & 0.71 \\
\hline \multirow[t]{3}{*}{ Pt-196 } & $2_{2}^{+}$ & [19] $-0.39(16)$ & -0.33 & -0.421 \\
\hline & $4_{1}^{+}$ & [19] 1.03 (12) & 1.007 & 1.018 \\
\hline & $2_{1}^{+}$ & [20] $0.42(12)$ & 0.44 & 0.490 \\
\hline \multirow[t]{2}{*}{ Pt-198 } & $2_{2}^{+}$ & - & -0.25 & -0.281 \\
\hline & $4_{1}^{+}$ & - & 0.38 & 0.422 \\
\hline
\end{tabular}

Hamiltonian (Equation (2) and Equation (11)) using NPBOS computer code. ${ }^{182-200} \mathrm{Pt}$ nuclei $(Z=78)$ have two proton bosons $\left(N_{\pi}=2\right.$ normal configuration) and the neutron boson varied from $N_{v}=13$ for ${ }^{182} \mathrm{Pt}$ isotope to $N_{v}=4$ for ${ }^{200} \mathrm{Pt}$ isotope.

The energy levels in Pt isotopes especially (ground state band) are a good agreement with the experimental data. In this study, we see that the excitation energy of the state $3_{1}^{+}$in ${ }^{186-200} \mathrm{Pt}$ isotopes (heavier Pt isotopes) is predicted sometimes too high in comparison with experimental data.

In the IBM-2-CM approach, the lightest Pt isotopes are deformed $\left({ }^{172-178} \mathrm{Pt}\right)$. In ${ }^{186} \mathrm{Pt}$ isotope a prolate shape and a $\gamma$-soft minimum coexist, but a well-deformed prolate minimum quickly develops in ${ }^{180} \mathrm{Pt}$ isotope, becoming the most pronounced prolate minimum at the mid-shell, i.e., in ${ }^{182} \mathrm{Pt}$ isotope with the prolate shape remaining well pronounced up to ${ }^{186} \mathrm{Pt}$ isotope. Moving towards heavier mass Pt isotopes, $\gamma$-flat energy surfaces start to develop. The ${ }^{198-200} \mathrm{Pt}$ isotopes tend to spherical shape because of the approach to magic number.

Concerning electric transition probability $\mathrm{B}(\mathrm{E} 2)$ values, we find that in all calculations the overall trend is reproduced reasonably very well in some transitions, but notice some discrepancies present case of the decay of the $0_{2}^{+}$states 
in heavier Pt isotopes, in general, better than the values calculated by Bjjker et al., [11].

\section{Conflicts of Interest}

The authors declare no conflicts of interest regarding the publication of this paper.

\section{References}

[1] Wood, J.L. (1982) Testing the Interacting-Boson and Interacting Boson-Fermion Approximations by the Study of Radioactive Decay Schemes with the Unisor on-Line Isotope Separator. In: Feng, D.H., Ed., Contemporary Research Topics in Nuclear Physics, Plenum Press, New York, 451-474.

https://doi.org/10.1007/978-1-4684-1134-8_26

[2] Heyde, K., Isacker, P., Van Waroquier, H., Wood, J.L. and Heyer, R.A. (1983) Coexistence in Odd-Mass Nuclei. Physics Reports, 102, 291-393. https://doi.org/10.1016/0370-1573(83)90085-6

[3] Heyde, K. and Wood, J.L. (2011) Shape Coexistence in Atomic Nuclei. Reviews of Modern Physics, 83, 1467-1521. https://doi.org/10.1103/RevModPhys.83.1467

[4] Garcia-Ramos, J.E. and Heyde, K. (2009) The Pt Isotopes: Comparing the Interacting Boson Model with Configuration Mixing and the Extended Consistent-Q Formalism. Nuclear Physics A, 825, 39-70. https://doi.org/10.1016/j.nuclphysa.2009.04.003

[5] Garcia-Ramos, J.E., Hellemans, V. and Heyde, K. (2012) Concealed Configuration Mixing and Shape Coexistence in the Platinum Nuclei. AIP Conference Proceedings, 1491, 109-112. https://doi.org/10.1063/1.4764214

[6] Puddu, G., Scholten, O. and Otsuka, T. (1980) Collective Quadrupole States of Xe, $\mathrm{Ba}$ and Ce in the Interacting Boson Model. Nuclear Physics A, 348, 109-124. https://doi.org/10.1016/0375-9474(80)90548-5

[7] Iachello, F. and Arima, A. (1987) The Interacting Boson Model. Cambridge University Press, Cambridge. https://doi.org/10.1017/CBO9780511895517

[8] Otsuka, T. and Yoshida, N. (1985) The IBM-2 Computer Program NPBOS. University of Tokyo, Tokyo.

[9] Duval, P.D. and Barrett, B.R. (1982) Quantitative Description of Configuration Mixing in the Interacting Boson Model. Nuclear Physics A, 376, 213-228. https://doi.org/10.1016/0375-9474(82)90061-6

[10] Duval, P.D. and Barrett, R. (1981) Shell-Model Description of the Interacting-Boson-Model Parameters for Two Non-Degenerate Shells. Physical Review Letters, 46, 1504-1507. https://doi.org/10.1103/PhysRevLett.46.1504

[11] Bijker, R., Dieperink, A.E.L., Soholten, O. and Spanhoff, R. (1980) Description of the Pt and Os Isotopes in the Interacting Boson Model. Nuclear Physics A, 344, 207-232. https://doi.org/10.1016/0375-9474(80)90673-9

[12] Singh, B. and Roediger, J.C. (2010) Nuclear Data Sheets for A=182. Nuclear Data Sheets, 111, 2081-2330. https://doi.org/10.1016/j.nds.2010.08.001

[13] Baglin, C.M. (2010) Nuclear Data Sheets for A=184. Nuclear Data Sheets, 111, 275-523. https://doi.org/10.1016/j.nds.2010.01.001

[14] Baglin, C.M. (2003) Nuclear Data Sheets for A=186. Nuclear Data Sheets, 99, 1-196. https://doi.org/10.1006/ndsh.2003.0007 
[15] Singh, B. (2002) Nuclear Data Sheets for A=188. Nuclear Data Sheets, 95, 387-541. https://doi.org/10.1006/ndsh.2002.0005

[16] Singh, B. (2003) Nuclear Data Sheets for A=190. Nuclear Data Sheets, 99, 275-481. https://doi.org/10.1006/ndsh.2003.0009

[17] Baglin, C.M. (2012) Nuclear Data Sheets for A=192. Nuclear Data Sheets, 113, 1871-2111. https://doi.org/10.1016/j.nds.2012.08.001

[18] Singh, B. (2006) Nuclear Data Sheets for A=194. Nuclear Data Sheets, 107. 1531-1746. https://doi.org/10.1016/j.nds.2006.05.004

[19] Huang, X.L. (2007) Nuclear Data Sheets for A=196. Nuclear Data Sheets, 108, 1093-1286. https://doi.org/10.1016/j.nds.2007.05.001

[20] Huang, X.L. (2009) Nuclear Data Sheets for A=198. Nuclear Data Sheets, 110, 2533-2688. https://doi.org/10.1016/j.nds.2009.09.002

[21] Kondev, F.G. (2007) Nuclear Data Sheets for A=201. Nuclear Data Sheets, 108, 365-454. https://doi.org/10.1016/j.nds.2007.01.004

[22] Xu, F.X., Wu, C.S. and Zeng, J.Y. (1989) Relations for the Coefficients in the I(I+1) Expansion for Rotational Spectra. Physical Review C, 40, 2337-2341. https://doi.org/10.1103/PhysRevC.40.2337

[23] Isacker, P., Van Heyde, K., Jolie, J. and Sevrin, A. (1986) The F-Spin Symmetric Limits of the Neutron-Proton Interacting Boson Model. Annals of Physics, 171, 253-296. https://doi.org/10.1016/0003-4916(86)90002-3

[24] Subber, A.R.H., Robinson, S.J., Hungerford, P., Hamilton, W.D., Isacker, P., Van Kumar, K., Park, P., Schreckenbach, K. and Colvin, G. (1987) The Level Structure of ${ }^{76} \mathrm{Se}$ and ${ }^{78} \mathrm{Se}$ and the Systematics of Selenium Isotopes within the Framework of the DDM. Journal of Physics G: Nuclear and Particle Physics, 12, 807-837. https://doi.org/10.1088/0305-4616/13/6/011

[25] Raman, S., Malarkey, C.H., Milner, W.T., Nester, C.W. and Selson, P.H. (1987) Transition Probability, $B(E 2) \uparrow$, from the Ground to the First-Excited $2^{+}$State of Even-Even Nuclides. Atomic Data and Nuclear Data Tables, 36, 1-96. https://doi.org/10.1016/0092-640X(87)90016-7

[26] Finger, M., Foucher, R., Husson, J.P., Jastrzebski, J., Johnson, A., Astner, G., Erdal, B.R., Kjelberg, A., Patzelt, P., Hoglund, Å., Malmskog, S.G. and Henck, R. (1972) Properties of Low-Lying Levels in the Even Platinum Nuclei $(182 \leq \mathrm{A} \leq 192)$. Nuclear Physics A, 188, 369-408. https://doi.org/10.1016/0375-9474(72)90064-4

[27] Eid, E. and Stewart, N.M. (1985) Studies of the Low-Lying Levels in ${ }^{192} \mathrm{Pt}$ and ${ }^{192} \mathrm{Os}$. Zeitschrift für Physik A Atoms and Nuclei, 320, 495-506. https://doi.org/10.1007/BF01415728

[28] Stelzer, K., Rauch, F., Elze, T.W., Gould, C.E., Idzko, J., Mitchell, G.E., Nottrodt, H.P., Zoller, R., Wallersheim, H. and Emling, H. (1977) Study of E2 Transition Probabilities in ${ }^{194} \mathrm{Pt}$. Physics Letters B, 70, 297-300. https://doi.org/10.1016/0370-2693(77)90662-1

[29] Glenn, J.E. and Saladin, J.X. (1968) Determination of the Static Quadrupole Moments of the First $2^{+}$States of ${ }^{194} \mathrm{Pt},{ }^{196} \mathrm{Pt}$, and ${ }^{198} \mathrm{Pt}$. Physical Review Letters, 20, 1298-1300. https://doi.org/10.1103/PhysRevLett.20.1298

[30] Bolotin, H.H., Stuchbery, D.L., Morrison, I., Kennedy, D.L., Ryan, C.G. and Sie, S.H. (1981) Lifetimes of Excited States in ${ }^{196,}{ }^{198} \mathrm{Pt}$; Application of Interacting Boson Approximation Model to Even Pt Isotopes Systematics. Nuclear Physics A, 370, 146-174. https://doi.org/10.1016/0375-9474(81)90759-4 